\title{
Eco-regional approaches, why, what and how
}

\section{R. RABBINGE}

Netherlands Scientific Council for Government Policy, P.O. Box 20004, 2500 EA The Hague, and Department of Theoretical Production Ecology, Wageningen Agricultural University, P.O. Box 430, 6700 AK Wageningen, and C.T. De Wit Graduate School for Production Ecology, Wageningen, The Netherlands

Key words: aggregation level, objectives, systems boundaries

Abstract. Eco-regional studies and approaches are more and more demanded by policy makers. They help to make strategic decisions and to explore options for development. The possibilities for such studies have increased as appropriate research tools became available, information could be collected and integration of disciplinary knowledge at various aggregation levels could take place through systems approaches.

The combination of detailed biophysical information, with farming systems research and with socio-economic studies on macro and meso levels enabled the development of explorative, predictive and analytic studies at regional level.

Thus, eco-regional research helps in priority setting at lower integration levels and may give an answer to questions formulated at the regional level. A solid scientific basis of these studies could be given by systems approaches at all integration levels that integrate detailed biophysical studies and socio-economic research.

\section{Introduction}

Eco-regional approaches may become the buzzword for the research agenda of the nineties and could easily be considered as old wines in new bottles. That would be a pity as eco-regional approaches may help to answer questions at the regional level and fulfil the promise of integration of disciplinary approaches which took place in the biophysical and socio-economic sciences. They may integrate studies at various integration levels, bridge the gaps between basic sciences and applied sciences, form an unifying problem oriented concept for the disciplinary contributions and create new rewarding research questions. Their possibilities as vehicle in understanding, gaining insight and exploring options for development are increasing as many new tools are becoming available.

A description of eco-regional approaches is given in this contribution, why they are so important, and how they can take place. 


\section{Eco-regional approaches, why and what}

The need to understand better how regional development and changes affect the possibilities and limitations of individuals in a region - farmers, other land users and other economic actors - is increasing. Studies at lower levels of integration than farm level cannot be generalised since they do not take into account changes in the environment at regional level that dictate the possibilities at lower integration levels. Land use studies and productivity studies at field or farm level often neglect the change in het biophysical and socio-economic environment in terms of constraints or new chances. Therefore the assumption that the environment is considered as an independent forcing variable is not true.

These changes may result in new constraints for example water availability and nutrient shortage or in new chances due to better possibilities to buy external inputs.

Eco-regions may be considered as systems with well defined boundaries within which farms and other elements and their interaction take place. The eco-regional approach enables the systematic study of (changes in) land use, and the study of agricultural systems within these systems. Biophysical and socio-economic knowledge and insight is further developed and integrated or synthesized to investigate and understand better how these systems function and how agricultural systems operate within these regions. Those studies are combined in eco-regional studies that explore possibilities at the regional level. Various objectives and constraints may be distinguished at the regional level that are completely different from the same characteristics at the farm level.

In the eco-regional approach a region is identified by its natural, administrative or socio-economic boundaries, within which the main rural and land developmental issues are made explicit. All important relations between agroecological systems and other forms of land use are specified, in particular those related to limited natural resources and limited socio-economic resources. In that way the eco-regional approach emphasizes the specific characteristics of a region. These specific characteristics help to identify the possibilities and constraints of these regions. Those constraints may be biophysical or socio-economic. The first may be dictated by climatic conditions and limited natural resources such as water and nutrient availability or external inputs or by lack of technological insight and knowledge. The latter by (skilled) labour, capital or insight in and availability of markets.

Biophysical and socio-economic objectives may play in all situations an important role. The need of studies at an eco-regional level has increased considerably as policy objectives and constraints are often defined at that level and determine the possibilities of farming systems.

Eco-regional studies are at the intermediate level between the farming systems approaches and the macro economic analysis that more and more 
consider the world as a global village. During the last decade many tools have become available that enable eco-regional approaches of a high quality. Geographical information systems (GIS) are used as appropriate tools for describing, analyzing and characterising eco-regional systems. Crop models may be used as integrating tools and explorative instruments to determine the potential and attainable yields. Many types of scenario approaches such as Interactive Multiple Goal Linear Programming (IMGLP) are becoming available with which various scenarios may be developed. Both help to derive options and to make general intentions and aims such as sustainable development operational.

\section{Eco-regional approaches, how}

The aim of the eco-regional approach dictates the methodology and the tools that should be used. Explorative studies aiming at the exploration of various possibilities are different from predictive studies that investigate what may be expected in the near future: which are again different from descriptive analytic studies that aim at understanding the interactions within the ecoregional system, and their response to changes in the environment.

In predictive and explorative studies a time horizon is needed. Predictive studies using the analysis of the present and contemporous development as main elements need a time horizon that is not too far away (less than 10 years). Trends and developments of the recent past dictate in those studies the possibilities and expectations for the near future The predictive studies are like weather forecasts. The closer the time horizon the more reliable the outcomes of such studies. Long term predictions are dangerous as they presume absence of discontinuities in trends. They may accept some variability in outcomes but always around a general trend. In predictive studies, trend analysis of certain and uncertain characteristics of systems play an important role. The reliability of forecasts (predictions) is determined by the accuracy of the input relations in connection with the environment. Plausibilities in those studies are measured as reliability of the prediction. Therefore probability is a good quality criterium for such studies.

There are many examples of such studies. The majority of eco-economic models for regions or for countries such as those developed by LEI-DLO, SC-DLO, Worldbank and many other institutions are used for these predictive analysis. The study of the Dutch Central Economic Planning Agency designs three scenarios, 'global shift, European renaissance and Balance Growth'. In each of these scenarios present developments are considered and amplified or cut back. The spectrum of possibilities is wide and shows the biotechnical, agro-technological or technical economic negative feedback are implicit in those studies and based on judgements of the authors. 
Explorative studies are of a completely different nature. They explore the future on basis of technical knowledge and normative and technical objectives and constraints. Technical knowledge is in most cases based on insight of the way biophysical systems operate. In agro-ecosystems such technical limits may be found in the amount of radiation arriving from the sun, the temperature and conservative characteristics of the systems such as the physiological traits that describe photosynthesis. Potential, attainable and actual production levels may be distinguished that are different in different production situations (Rabbinge 1993).

It is the task of biophysical research to identify how growth and yield defining factors can be used at field but also at higher integration levels and how they can be affected in such a way that in technical terms optimal crop and cropping systems are developed. In technical terms means in this case that the efficiency of the external inputs per unit of product is maximized or that the emission of nutrients per unit of product is minimized. It may be desirable that for ecological reasons the emission of nutrients and immission of pesticides per unit of area should be minimized and that would mean in good production situations a suboptimal production technique in terms of efficiency per unit of product for the other inputs.

Thus the biophysical sciences enable an explicit discussion on what preference should be given to various objectives. It shows also that some ecological aims are within the ecological domain conflicting and not conflicting with socio-economic aims. It is the contribution of biophysical sciences to ecoregional studies to design, production technologies, to identify and quantify the various aims and the possibilities to reach those objectives, to gain better insight in agro-ecosystems and to use that in the improvement of those systems.

\section{Examples of eco-regional approaches}

Demonstrations of explorative studies in this book are given by Penning de Vries et al. (1995), Van Latesteijn (1995), and Aggarwal et al. (1995).

The study of Van Latesteijn (1995) is explorative and shows how various objectives (socio-economic, eco-logical and agri-cultural) can be achieved by optimizing land use. Various scenarios of land use depending on the relative weight of various objectives are developed. The study of Penning de Vries $e t$ al. (1995) is also explorative but of a different nature. The biophysical limits to feed the world population by various production systems are investigated under potential and water limited circumstances. That type of study was also done some twenty years ago (Buringh et al. 1975). However, the explorative studies of Penning de Vries et al. (1995) take water availability, production technologies and required food in terms of different diets into account. More- 
over, a more detailed land evaluation using grids and dominating biophysical soil and climate characteristics is used.

The results show that there are wide margins on a world scale and considerable differences between various regions. In some regions 'anything goes', in other regions all means are necessary to attain sufficient food for the population in those areas. The possibilities to feed a world population with different technologies and diets vary between $11 \cdot 10^{9}$ to $44 \cdot 10^{9}$ capita. In the latter case, still only $30 \%$ of the world's area is in use for agriculture that is maintained in a sustainable way.

The danger of these explorative studies to overestimate the real possibilities to achieve the potentials can not be denied. However, studies of a predictive nature tend to overestimate negative developments such as soil degradation. Studies of the Worldwatch Institute predict that $5 \cdot 10^{9}$ humans are too much for the carrying capacity of the world of Brown et al. (1993). These studies indicate that $2 \cdot 10^{9}$ human beings are more or less the maximum carrying capacity of mother earth.

The considerable differences between predictive and explorative studies may help to gain understanding in how to intervene. For that reason, analytic knowledge at various integration levels is necessary. Institutional constraints may prevent a proper use of the possibilities and limit the use of adequate policy instruments. To identify such constraints and to enable interventions that may help to reach the desired explored perspectives, good knowledge of farm systems and the way they function in regions is needed.

In that way explorative, predictive and descriptive/analytical studies are complementary. They may stimulate each other and through synergism help to stimulate regional development.

Examples of eco-regional approaches that aim at prediction are found in many programs of the CGIAR institutes. The eco-regional initiatives in West Africa and the Andean highlands are at present dominated by predictive analysis of the changes in land use. They show the devastating effects of a continuation of such developments. It is for that reason that there is an increasing demand for explorative studies that complement these discouraging explorative analysis. A detailed and appropriate analysis of the way the various actors function in eco-regional systems may help to design efficient and effective interventions that bring explorative possibilities more in line with predictive dystopia.

\section{Linkages between various integration levels}

Tools such as crop modelling, GIS, and IMGLP techniques have become well developed and mature instruments that enable problem oriented eco-regional approaches. 
Systems approaches have already been used to identify knowledge-based management strategies to improve resource use management at the crop level with a minimum negative environmental impact of Rabbinge (1993). Tools such as crop simulation models, that can predict the yields attainable in a range of environments, have been developed to generate technologies targeted at specific local environments by Van Latesteijn (1995).

Constraints for growth in productivity stem from biophysical and socioeconomic characteristics at regional levels including water resources. Systems approaches should therefore be extended to regional levels in order to identify the relevance of these constraints and to develop solutions. Options for land use at regional levels that meet region-specific goals must incorporate spatial information on environmental and socio-economic constraints. Several tools, such as GIS and land evaluation systems are used, and new methodologies of Penning de Vries et al. (1995) have been developed to integrate agro-ecological and socio-economic approaches at regional levels. Although spatial information is often incomplete, an eco-regional approach is meant to integrate that information, determine lacks of knowledge and to make the knowledge and insight operational. The eco-regional approach to problems in agriculture is schematically presented in figure 1.

The relationships between components of production systems and the environment must be studied to understand better the behaviour of the systems. Knowledge from studies at the level of genome, plants and crops of water and nutrient use, of consequences of pests, and of effects of alternative management techniques are integrated and engaged to define production technologies tailored to specific needs and opportunities. Implications of different scenarios for environmental changes and improved production systems, can be made visible by using optimization techniques. Socio-economic objectives, constraints and analyses are an important part of such optimizations. Depending on the priority given to various objectives such as cost minimization, employment, or use of natural resources, the results of the scenarios are very different.

The eco-regional approach makes explicit the choices for various ways of agricultural land use that can be made, and the unavoidable trade offs between objectives. The new insights can also be used to prioritize crop research and to identify needs for additional biophysical and socio-economic information. The various links are presented in figure 2.

These studies are still characterized by explorative analysis and not so much by realistic designs for future developments. That may only be possible when a synthesis between the various approaches is operational. Another advantage of this approach is that detailed biophysical research at crop or cropping systems level is focused and integrated to higher aggregation levels.

The detailed research projects and questions at the lower level are derived from the higher aggregation level. By an interative way of working priority 


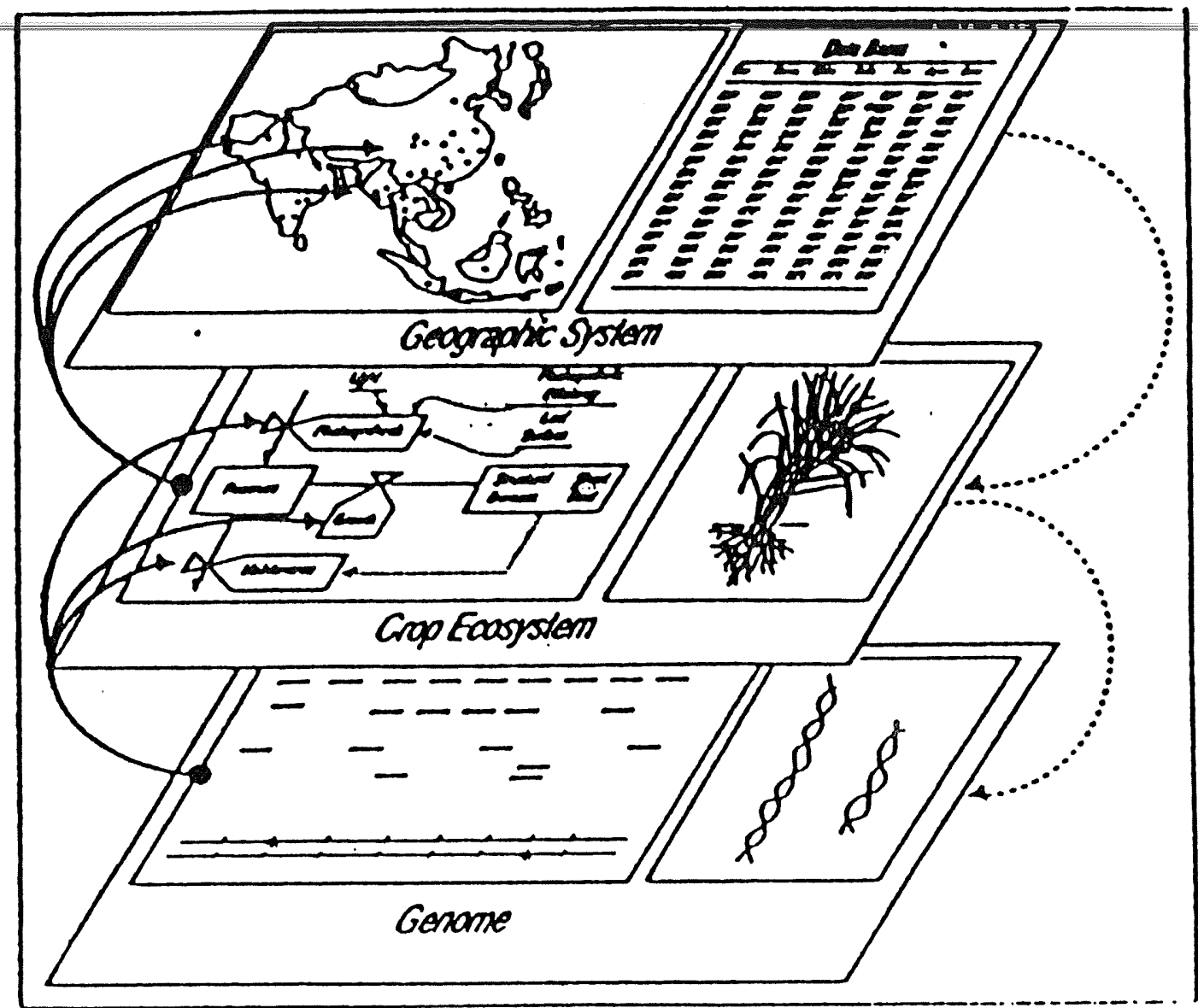

Fig. 1. Systems approaches to link agricultural research activities at different scales (source: IRRI 1994).

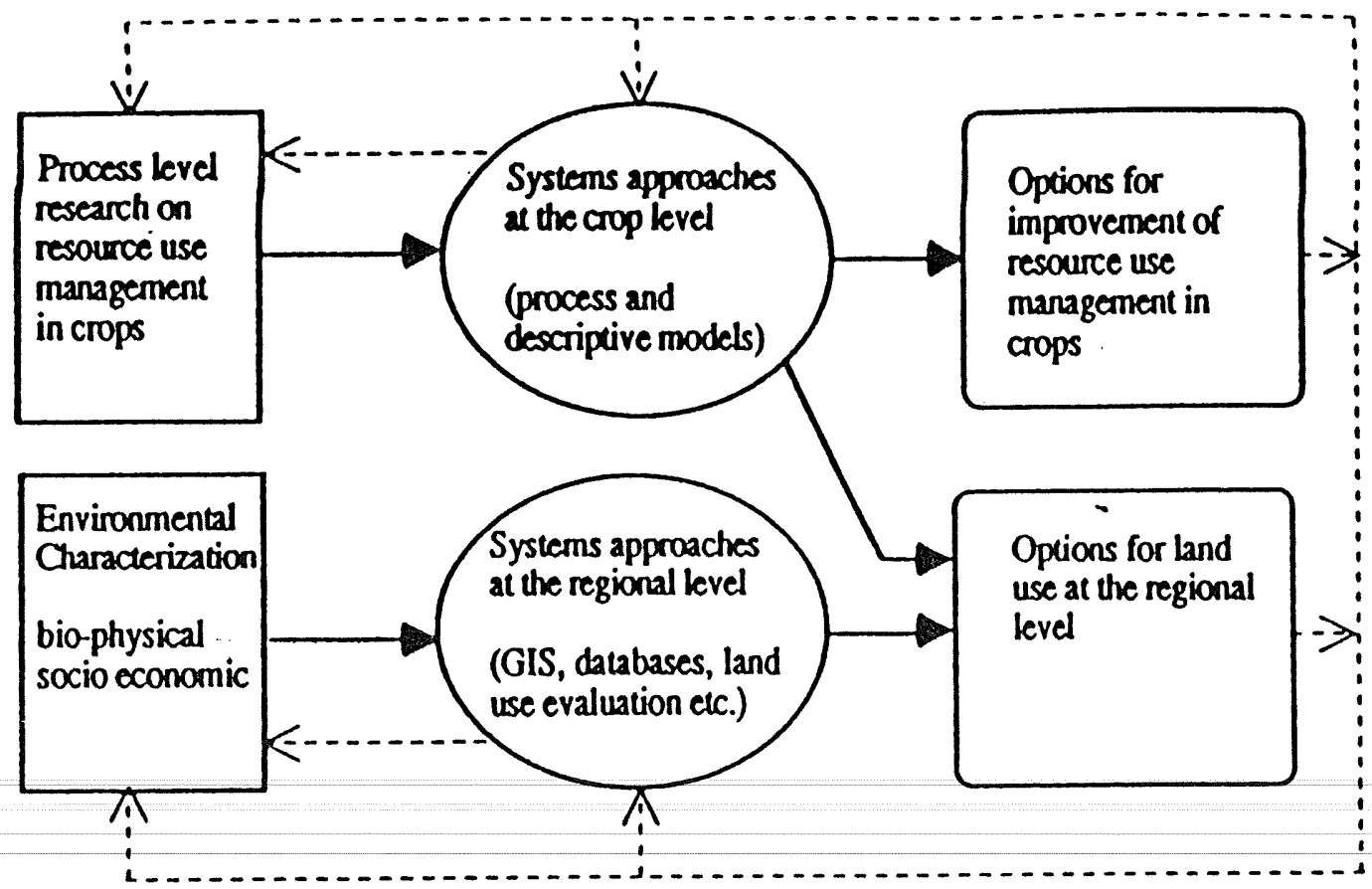

Fig. 2. Research of structure eco regional networks. Dotted lines indicate feed-back information. 
setting in detailed process research is determined by the problem solving synthesizing research activities at higher levels. The model activities at the regional level are in this example of an explorative nature.

\section{Perspectives for eco-regional approaches}

The perspectives of eco-regional approaches are bright. They may fulfil various expectations such as:

- problem oriented research;

- take into account the biophysical and socio-economic characteristics of regions;

- integrate contributions of various disciplines;

- are wide without loosing deepness;

- enable the operationalisation of vague intentions such as sustainable agriculture;

- demonstrate how resource use efficiency may be optimized at regional levels.

There are, however, also some dangers in the eco-regional approach:

- they may be restricted to methodologies;

- they may lead to too much tool orientation losing the problem orientation;

- they may lead to delay of interventions and may be used as an alibi for not doing things.

It is the task of the CG-institutes, the NARS and advanced systems groups to use the chances and to prevent a development away from problem-oriented research. In this book the various tools and institutional requirements will be discussed. Ultimately that should lead to an improved collaboration and to problem solving at regional level. Eco-regional studies are no aim in themselves, they should be used for a better guidance of regional development within the context of a global rapidly changing environment.

\section{Acronyms}

CGIAR Consultative Group on International Agricultural Research

GIS

IMGLP

Geographical Information System

NARS

Interactive Multiple Goal Linear Programming

National Agricultural Research System 
References

Aggarwal P K, Kalra N, Bandyyopadhyay S K, Selvarajan S (1995) A systems approach to analyze production options for wheat in India (pages 166-186 of this volume).

Brown, L R, Durning A, Falvin C, et al. (1993) State of the World. W.W. Norton Company, New York, USA.

Buringh, P, Van Heemst H D J, Staring G J (1975) Computation of the absolute maximum food production of the world. Dept. of Tropical Science, Wageningen Agricultural University, Wageningen, The Netherlands.

IRRI (1994) Mid term plan. International Rice Research Institute (IRRI), Los Baños, Philippines.

Van Latesteijn H C (1995) Scenarios for land use in Europe: agro-ecological options within socioeconomic boundaries (pages 43-63 of this volume).

Penning de Vries F W T, Van Keulen H, Rabbinge R (1995) Natural resources and limits of food production in 2040 (pages 65-87 of this volume).

Rabbinge R (1993) The ecological background of food production. Pages 2-29 in Crop protection and sustainable agriculture (Ciba Foundation Symposium 177), Wiley, Chichester, UK. 